Asociación de Jóvenes Historiadores y Arqueólogos de Murcia

PANTR REL

REVISTA DE CIENCIA

$$
\begin{array}{r}
Y \\
\text { DIDÁCTICA } \\
\text { DE LA HISTORIA } \\
\text { III - } 2^{2} \text { época }
\end{array}
$$




\section{ASOCIACIÓN DE JÓVENES HISTORIADORES Y ARQUEÓLOGOS DE MURCIA}

\section{PANTA REI. REVISTA DE CIENCIA Y DIDÁCTICA \\ DE LA HISTORIA III. $2^{a}$ época}


CONSEJO EDITORIAL: LAURA ARIAS FERRER

ALEJANDRO EGEA VIVANCOS

ANTONINO GONZÁLEZ BLANCO

RAFAEL GONZÁLEZ FERNÁNDEZ

JOAQUÍN LOMBA MAURANDI

ENRIQUE QUINTANA CIFUENTES

GONZALO MATILLA SÉIQUER

JOSÉ ANTONIO MOLINA GÓMEZ

REDACTOR JEFE:

ÁNGEL LUIS GONZÁLEZ TORRES

Depósito legal: MU-966-1995

I.S.S.N. 1136-2464

Edición de Compobell, S.L. Murcia 


\section{ÍNDICE}

AGRADECIMIENTOS

Presentación

Por Alejandro Egea Vivancos, Laura Arias Ferrer

\section{ARTÍCULOS}

Análisis microscópico de la industria lítica: la traceología

Por Ignacio Martín Lerma

Reflexiones en torno al estudio de la economía en Prehistoria

Por Valentín Martínez García

Aplicaciones SIG en el análisis de las sociedades del pasado. Un caso de estudio: Las primeras comunidades campesinas del Levante Peninsular

Por Gabriel García Atiénzar

La situación actual de los estudios de egiptología en España

Por José Javier Martínez García

La vida cotidiana en el Imperio Asirio. Usos y costumbres de un pueblo que conquistó las Cuatro Regiones del Mundo

Por Ángel Luis González Torres

Las formas de intercambio y las estructuras comerciales orientalizantes en la Vega Baja del Segura: dos variables de estudio arqueológico

Por Sara Pernas García 
Evolución de los patrones de asentamiento en época ibérica. Una propuesta de estudio del mundo ibérico murciano a través del análisis del poblamiento

Hispania Tierra de Roma. Organización y gestión del suelo

Por $M^{a}$ Carmen Santapau Pastor

Hacia una arqueología de la España bizantina. Breves notas a propósito del seminario Work in progress

Por Jaime Vizcaíno Sánchez

Reflexiones acerca de un estudio sobre las estancias auxiliares en la arquitectura barroca catedralicia

Por Francisca del Baño Martínez

La investigación sobre fisiognomía y expresión de las pasiones. Objetivos y metodología

Por María del Mar Albero Muñoz 


\title{
REFLEXIONES ACERCA DE UN ESTUDIO SOBRE LAS ESTANCIAS AUXILIARES EN LA ARQUITECTURA BARROCA CATEDRALICIA
}

\author{
FRANCISCA DEL BAÑO MARTÍNEZ ${ }^{1}$
}

\section{Resumen}

Aquí se recogen algunas de la reflexiones acerca del un trabajo de investigación dedicado al análisis de los espacios de la catedral que no corresponden propiamente a la zona del templo, pero que han desarrollado una función imprescindible en el conjunto catedralicio, tanto en lo que se refiere a los aspectos litúrgicos, como a los de tipo institucional. Se trata de un estudio en el que los principales ámbitos objeto de interés han sido las sacristías, salas capitulares, archivos, bibliotecas, claustros y contadurías. A partir de su análisis se ha pretendido indagar en el mecanismo interno de una catedral en la época mencionada, reflexionando sobre las cualidades y características de estos espacios, ya que eran los que estaban destinados a favorecer su funcionamiento.

Palabras clave: Barroco, Arquitectura, catedral, sacristía, sala capitular.

\begin{abstract}
The present work has been devoted to the analysis of the additional rooms which together with the temple constitute a cathedral. These rooms have had an essential function not only in relation to the liturgy but also in relation to institutional aspects. Thus, sacristies, chapter rooms, archives, libraries, cloisters and accountant

1 Este trabajo ha sido realizado en el seno del Proyecto de Investigación financiado por el Ministerio de Educación y Ciencia: «Reflejo de los nuevos cultos y devociones en las catedrales españolas durante el Barroco: Arquitectura, Arte y devoción». Ref. HUM2006-12319.
\end{abstract}


offices have been the main subjects of this work. Thanks to their study, the internal mechanism of cathedrals during that particular time has been investigated; we have reflected on the qualities and characteristics of these rooms since they were aimed at favouring their functioning.

Key words: Baroque, Architecture, cathedral, sacristies, chapter room.

En las páginas siguientes voy a tratar de resumir las principales ideas y objetivos de la labor de investigación que ha constituido mi tesis doctoral, el plan de trabajo y la metodología empleados, las distintas partes de que consta este estudio, las dificultades u obstáculos que han ido surgiendo durante el proceso, así como algunas de sus conclusiones más relevantes.

Bajo el título de la tesis doctoral «Estancias de uso y representación al servicio de las catedrales españolas durante el Barroco» se encuentra un trabajo de investigación dedicado al estudio de esos espacios de la catedral que no corresponden propiamente a la zona del templo, pero que han venido desarrollando una función imprescindible en el conjunto catedralicio, tanto en lo que se refiere a los aspectos litúrgicos, como a los de tipo institucional. De este modo, los principales ámbitos objeto de interés han sido las sacristías, salas capitulares, archivos, bibliotecas, claustros y contadurías.

La idea de realizar una tesis doctoral sobre este tema, surgió al amparo de un Proyecto de Investigación I+D de ámbito nacional, ${ }^{2}$ dirigido y coordinado por el Dr. Germán Ramallo Asensio, a su vez director de este trabajo. Dicho proyecto estaba centrado en el estudio de los cambios y transformaciones que se experimentaron en las catedrales españolas durante el periodo barroco, de tal modo que se entendió la dotación y/o renovación de estancias auxiliares, como una variante más en este sentido.

Uno de los principales objetivos marcados era el de llegar a comprender las catedrales españolas de los siglos XVII y XVIII, desde un punto de vista distinto al que ha predominado tradicionalmente en los estudios realizados sobre estos grandes edificios. Así, la atención no debía centrarse en el espacio del templo, donde se desarrolla el culto, sino que debía dirigirse al entramado de construcciones que han ido surgiendo en torno a él para distintos usos y servicios. A partir del estudio de estos ámbitos, se buscaba conocer el mecanismo interno de una catedral en la época mencionada, así como reflexionar sobre las propias características de estos espacios, ya que eran los que estaban destinados a favorecerlo.

2 «El comportamiento de las catedrales españolas del Barroco a la Restauración». Ministerio de Ciencia y Tecnología. Ref. BHA200-0192-C08-03. 


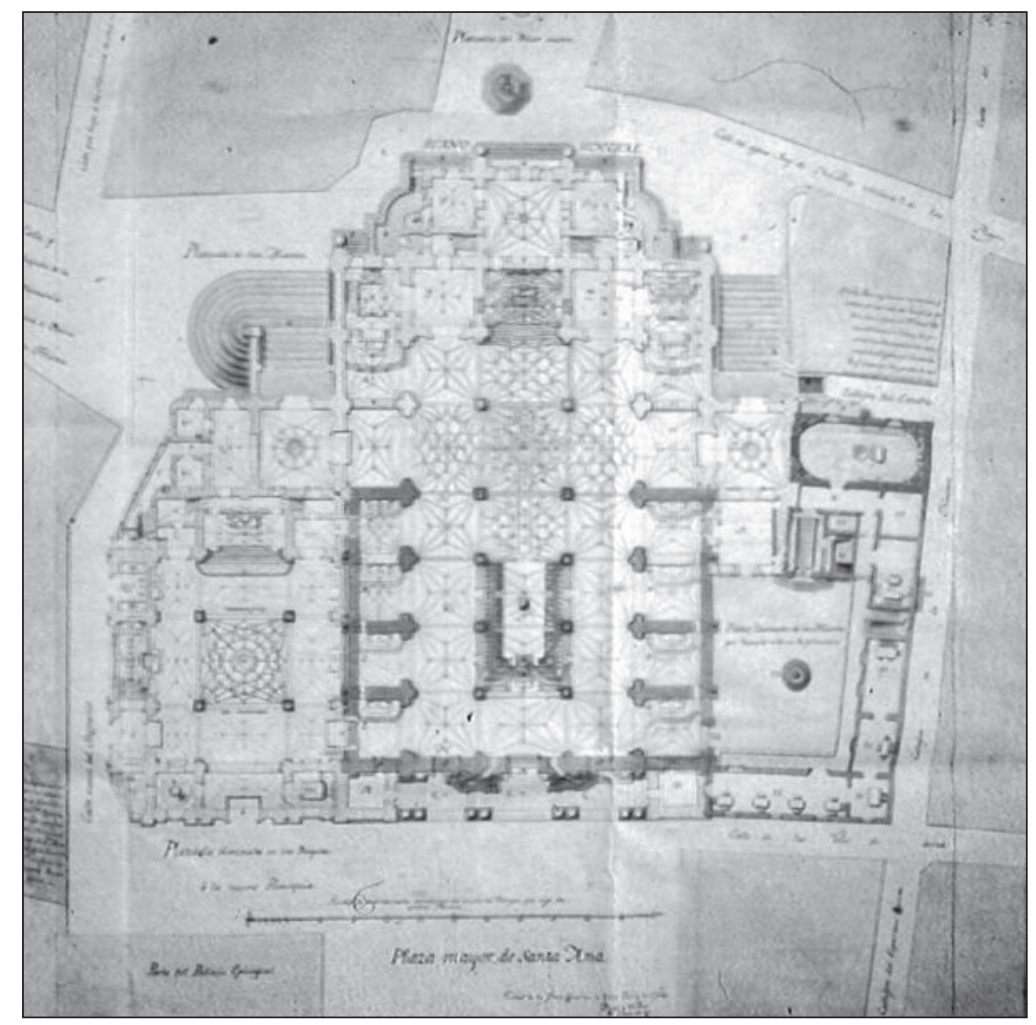

Figura 1. Plano de catedral de las Palmas de Gran Canaria, realizado en el siglo XVIII. En él aparecen representadas las distintas estancias auxiliares que se disponen en torno al templo.

Ante todo, no se podía olvidar un hecho incuestionable y es que la catedral es un edificio vivo, en el que el Cabildo necesita su espacio y esas necesidades espaciales han ido cambiando a través del tiempo en función de diversas circunstancias. Precisamente, esa misma versatilidad de uso y función que han presentado tradicionalmente algunas de estas estancias de la catedral, ha sido uno de los factores que más ha dificultado la realización de este trabajo.

\section{Principales dificultades surgidas en el transcurso de la investigación}

Ya desde los mismos inicios de esta investigación, ésta no se anunciaba como una tarea fácil, sino que se evidenciaban una serie de factores que la dificultarían considerablemente. En primer lugar se encontraba la enorme amplitud del tema y la gran dispersión geográfica que presentan las distintas catedrales del país, lo que imposibi- 
litaba la conveniente visita y búsqueda documental de cada una de ellas. A esto hay que añadir, además, que no siempre se ha podido tener acceso a la documentación contenida en sus archivos, pues algunos de ellos han permanecido cerrados durante todo el desarrollo de la tesis.

Como ya hemos adelantado previamente, otra de las grandes dificultades a la que nos hemos tenido que enfrentar en el transcurso de este trabajo, ha sido el frecuente cambio de uso o función que han sufrido muchas de las estancias catedralicias en las que se ha centrado el interés, algo que no siempre ha quedado convenientemente documentado. Esta circunstancia ha llegado a impedir, en determinadas ocasiones, que se pueda conocer el aspecto y la distribución original que presentaron algunas estas fábricas durante el periodo barroco. En otras, en cambio, se ha logrado una buena aproximación a dicha realidad a partir de los datos extraídos de fuentes bibliográficas antiguas, de documentos en los que se incluyen detalladas descripciones e, incluso, de interesantes planimetrías que se han conservado sobre este asunto.

Los continuos cambios y adaptaciones espaciales que se han sucedido en estos lugares con cierta recurrencia hasta la actualidad, alcanzaron probablemente uno de sus momentos culminantes entre los años finales del siglo XIX y los primeros de la siguiente centuria. Fue por entonces cuando se extendió una tendencia que caracterizó a muchas de las intervenciones «restauradoras» del momento, debido a la cual se llegó a despojar a algunas catedrales de varias construcciones de este tipo que se encontraban adyacentes al templo, siempre con la intención de poder mostrar así su volumen totalmente exento, tal y como ocurrió en el caso paradigmático de la catedral de León. Otra vertiente de esta misma moda se encaminó, en cambio, a desprender a las catedrales de su imagen barroca, al pretender devolverles un aspecto medieval mediante actuaciones como las que tuvieron lugar en las catedrales de Valencia, Urgell o Bilbao.

Posteriormente, hacia mediados del siglo XX, muchos de los espacios capitulares fueron integrados en los recorridos museísticos de sus respectivas sedes, desvirtuándolos así de las funciones y servicios para los que habían sido creados. Algunas acciones de este tipo llegaron a afectar seriamente no sólo a la naturaleza y distribución de estos espacios catedralicios, sino también al mobiliario que se encontraba contenido en su interior. Así, por ejemplo, la sillería de la sala capitular de la catedral de Murcia, a pesar de ser una obra de buena talla y factura barroca, fue reconvertida en vitrinas en las que exponer las piezas de orfebrería del museo catedralicio, inaugurado en el año 1957.

Finalmente a todos esos obstáculos que se han señalado, hay que añadir el hecho de que el conjunto de las estancias auxiliares en las catedrales continúa siendo una de las partes más desconocidas de la catedral. Esto se debe a que son aún realmente pocos los estudios e investigaciones que se han dedicado a ello, probablemente por 
la escasez, e incluso ausencia, de documentación que pueda aportar luz a este problema.

Tal vez por todas estas razones y al considerar las limitaciones que de todo ello se derivaban, se advirtió que no se podía realizar un estudio en el que se analizase con total precisión lo realizado, sino que se debía concebir dicha tesis doctoral como un primer paso a la gran tarea investigadora que se abre en torno a este tema. Se planteó, por lo tanto, como una aproximación o un incipiente marco teórico en el que poder encuadrar posteriormente otros estudios más concretos y profundos.

\section{Plan de trabajo y propuesta metodológica}

El plan de trabajo constó de varias fases que se podrían sintetizar del siguiente modo. En un primer lugar se procedió a reunir y revisar todo lo que se había publicado sobre estos espacios, tanto en la amplia bibliografía existente que versa sobre las catedrales españolas y en revistas especializadas, como en otras fuentes de información como pueda ser la red. Se pudo ver que en la historiografía tradicional, las alusiones a este tipo de dependencias son habitualmente escasas, confusas, y en muchas ocasiones incluso ausentes. De este modo, a pesar de que se encontraron referencias a estos espacios en distintas publicaciones analíticas e historiográficas, se llegó a la conclusión de que el interés por el estudio de estos recintos resulta bastante insólito. Así, se han podido ver reflejados frecuentemente de un modo indirecto, pero han sido muy contadas las ocasiones en las que han sido objeto de un tratamiento de carácter monográfico o exclusivo. ${ }^{3}$

Por lo tanto, tras el contraste de fuentes bibliográficas, se pudo ver que aún seguía siendo necesario concretar e identificar de forma pertinente algunas de las estancias, aclarando denominaciones, e incluso fijando cronologías. Para ello, ha resultado realmente valiosa la información obtenida de la consulta de una gran cantidad de documentos procedentes de diversos archivos catedralicios, diocesanos, provinciales e incluso municipales, sin olvidar otros de ámbito más general como el Histórico Nacional, el de Simancas, o el Secreto del Vaticano. Asimismo, también han servido de gran ayuda los textos de los estatutos y constituciones que fueron redactados con el fin de regular la actividad y el funcionamiento de las distintas sedes, sin olvidar las entrevistas realizadas a determinadas personas vinculadas a la vida catedralicia, verdaderos conocedores y moradores habituales de su espacio, quienes incluso ofrecieron notables referencias históricas. De forma paralela a este proceso, se iban rea-

3 En este sentido, en nuestro país destacan los estudios realizados por Eduardo Carrero Santamaría, quien ha realizado una serie de trabajos centrados en el análisis de estas dependencias catedralicias de la zona septentrional de España, fundamentalmente sobre el periodo medieval. 
lizando las pertinentes visitas a los distintos objetos de estudio. Finalmente, con los resultados obtenidos, se pudo pasar a realizar el análisis de estos recintos, intentando dar respuesta a los diversos interrogantes que iban surgiendo constantemente en torno a ellos.

Para la organización del trabajo, se consideró adecuado proceder a una distribución en dos grandes bloques. De este modo, el primero de ellos se dedicaría por completo a la sacristía catedralicia, con una primera parte teórica centrada en su análisis y una segunda constituida por un catálogo de los ejemplos más representativos. En el otro bloque nos ocuparíamos del estudio del resto de los espacios capitulares en la catedral, siguiendo para ello una estructura similar a la marcada en el caso de la sacristía.

El primer propósito marcado lo constituyó el análisis de los numerosos ejemplos de edificaciones que fueron construidas durante el periodo mencionado, así como también de aquéllas otras que sufrieron importantes reformas o remodelaciones. Pero pronto se advirtió que si únicamente se señalaban en este estudio los proyectos que, de un modo más o menos ambicioso, fueron llevados a la práctica, probablemente no se podría llegar a ofrecer una idea lo suficientemente aproximada del alcance que tuvo el fenómeno constructivo y modernizador de las estancias capitulares catedralicias durante el periodo barroco. Por ese motivo, se consideró necesario hacer referencia además a los numerosos proyectos que quedaron plasmados en el papel, e incluso a algunos otros que ni tan siquiera llegaron a manifestarse en dicho soporte, siendo alimentados únicamente por los deseos y expectativas de los miembros de las corporaciones capitulares.

En cuanto al marco cronológico seleccionado para delimitar este trabajo, se quiere hacer constar el hecho de que éste no se encuentra prefijado en absoluto de una manera rígida, pues a pesar de que el estudio se centra fundamentalmente en el estilo barroco, se ha hecho necesario incluir además ejemplos pertenecientes a periodos anteriores, por considerar que actuaron como precedentes de los que constituyen el objeto principal de la tesis. También sucede lo mismo con otros pertenecientes a la segunda mitad del XVIII, cuyos caracteres estilísticos y formales trascienden claramente el lenguaje barroco, pero que, a pesar de ello, vinieron a ofrecer una solución a necesidades planteadas con anterioridad. Por lo tanto se puede decir que continuaron con la misma dinámica constructiva que se había experimentado en el momento inmediatamente anterior, respondiendo a unas motivaciones similares y siendo auspiciados por unas mismas intencionalidades.

\section{Partes de que consta el estudio}

Una vez concretadas ya las pautas por las que se debía regir la labor investigadora, delimitados los objetos de estudio y conforme al plan de trabajo propuesto, se 
inició un proceso que duraría cinco años hasta la conclusión de la tesis doctoral. En ella, el primer capítulo se dedica al análisis de la sacristía mayor, la cual constituye una de las partes más nobles del edificio eclesiástico en general y del catedralicio en particular. Allí se prepara todo lo que concierne al acto litúrgico, desde los diversos elementos que componen su rico y abundante ajuar, hasta los vestidos de los oficiantes, los libros, velas, etc. En una primera parte teórica se atiende a diversos aspectos como la etimología de su denominación, su evolución a través de distintos periodos históricos, su ubicación en el conjunto de la catedral, los diferentes tipos existentes,

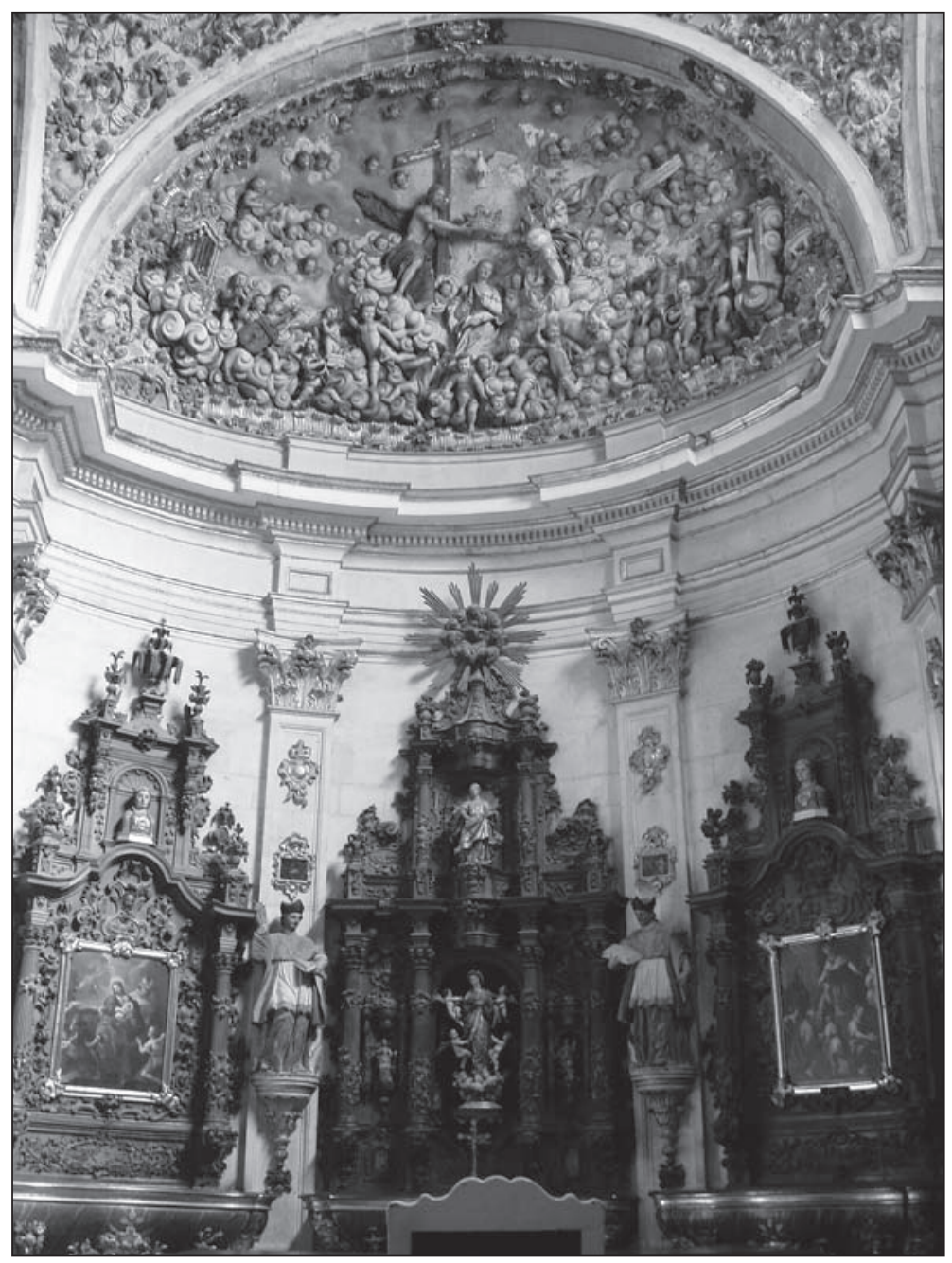

Lámina 2. Sacristía barroca de la catedral de Burgos. 
así como sus diversos usos y funciones, etc. Posteriormente se procede a la clasificación de los diversos ejemplos por orden cronológico y también en función de sus diferentes tipologías. De este modo quedaron distribuidos en tres secciones que se corresponden con los siglos XVI al XVIII.

El segundo bloque o capítulo queda enfocado al estudio de los espacios capitulares de la catedral, comenzando por la sala en la que se celebran las asambleas del cabildo. En ella se incluye también una primera sección teórica, que se ocupa de su funcionalidad, disposición, ubicación, ornamentación y a los rasgos formales que la caracterizan. Estos hacen que constituya el lugar idóneo de la catedral en el que el Cabildo celebra sus reuniones o asambleas. En ella también se celebran otros actos de gran importancia y solemnidad para la vida catedralicia, como son la toma de posesión de cargos u oficios, las elecciones entre candidatos a prebendas y dignidades, el nombramiento y recibimiento a nuevos prelados, etc. La segunda parte se dedica al análisis de los ejemplos más representativos, los cuales se organizan conforme a su cronología, salvo en el caso de las salas capitulares ovaladas, que por el hecho de constituir un tipo muy específico y muy poco habitual, se estudian en su conjunto.

Además de ello, se atiende también al proceso de remodelación que sufrieron las estructuras claustrales durante los siglos XVII y XVIII, así como al análisis de las distintas oficinas catedralicias. Entre ellas se encuentran las contadurías, archivos o

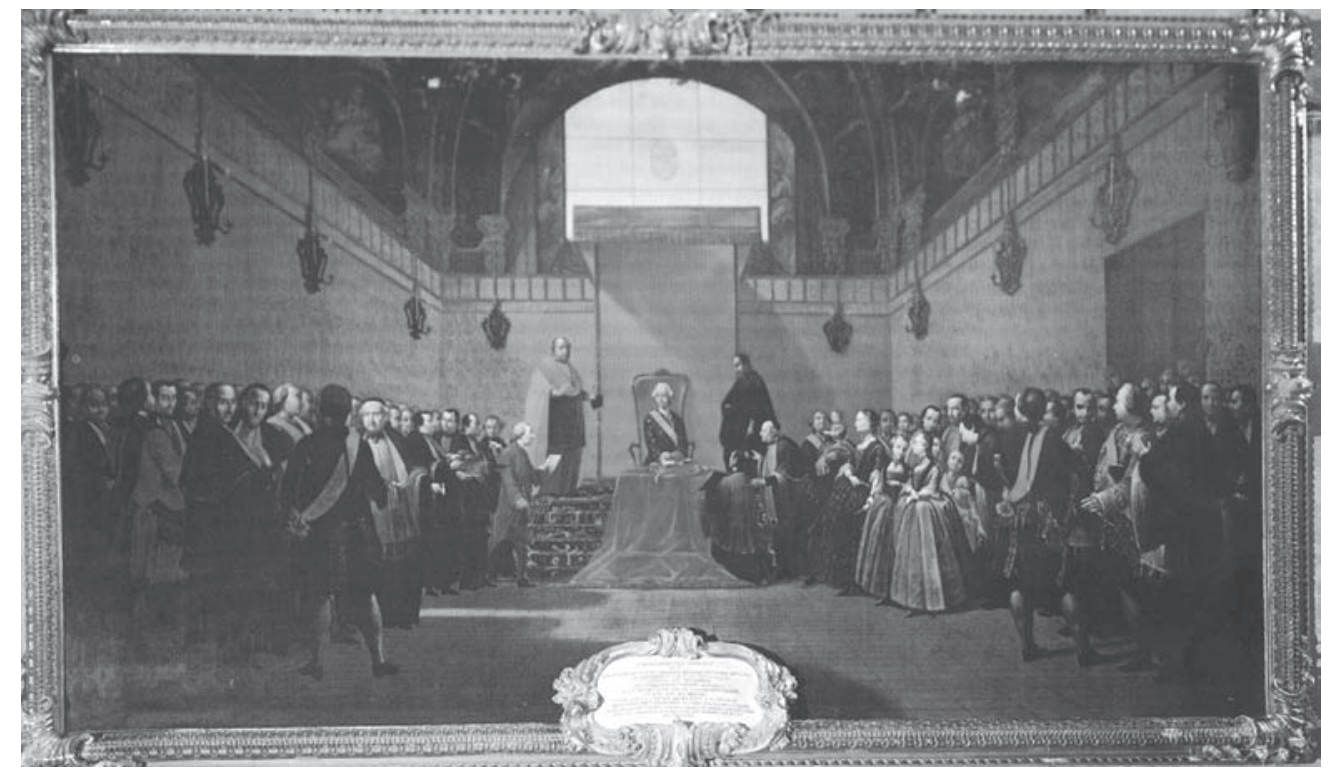

Figura 3. Cuadro en el que se representa a Carlos III tomando posesión de una canonjía en la sala capitular de la catedral de Barcelona. Manuel Tramulles, ca. 1770. 
también las bibliotecas. Se trata de ámbitos eminentemente funcionales, administrativos o de otra naturaleza, que eran destinados a atender a las necesidades cotidianas de la sede. Pero en ocasiones, su carácter práctico y funcional, no iba reñido en absoluto con el gusto barroco por la ornamentación y el detalle.

Del conjunto de estos espacios destaca la relevancia y el desarrollo adquirido por los archivos tras el Concilio de Trento. A partir de ese momento se dio un fuerte impulso a la creación de espacios específicos destinados a la custodia y salvaguarda de los documentos importantes de la sede, iniciándose una verdadera política archivística que anduvo paralela a la que se estaba gestando por entonces en el ámbito civil.

También se recoge la creciente importancia de los espacios destinados a la consulta y conservación de libros, no sólo religiosos, sino también de otras materias como el Derecho, la Gramática, Medicina, Literatura... El gran auge de estos espacios tuvo lugar tras el desarrollo de la imprenta, pero también se ha podido comprobar que en los siglos XVII y XVIII se continuaron edificando interesantes bibliotecas en nuestras catedrales.

Finalmente se atiende a otro de los espacios que adquirió un gran protagonismo en el conjunto de las estancias capitulares, como fue la contaduría, lo que, sin duda alguna, estuvo vinculado a un notable aumento de las rentas y con ello de las operaciones de recaudación, dotación o distribución de fondos.

\section{Conclusiones más relevantes}

Del estudio de los diferentes espacios de uso y representación que fueron dispuestos para el servicio de las catedrales durante los siglos del barroco, se desprenden una serie de conclusiones que cabe sintetizar muy brevemente en este momento.

La primera es un hecho evidente y es que una catedral no está formada por un único edificio: el templo, sino por un conjunto de edificaciones como son las salas capitulares, sacristías, claustros, oficinas, zonas de almacenaje, capillas, etc., las cuales frecuentemente han sido construidas en diferentes periodos y, por lo tanto, a pesar de estar supeditadas a un conjunto, mantienen una cierta autonomía.

De este modo, todas y cada una de estas edificaciones han sido creadas para dar respuesta a unas necesidades concretas y para desarrollar una funcionalidad precisa, constituyendo así las distintas partes integrantes un complejo organismo: la catedral, la cual ocupaba un lugar fundamental en el trazado urbano de las ciudades y en el entramado social del Antiguo Régimen, dado que además de sus propia misión pastoral y litúrgica, tenía una gran peso económico, cultural e incluso político.

En esos espacios funcionales anexos al templo desarrollaban sus tareas muchas de las personas que estaban vinculadas a la catedral, de tal modo que constituían ám- 
bitos en los que habitualmente se registraba una gran actividad y un cierto trasiego, muy diferentes a la quietud que domina en ellos en la actualidad.

Tras la labor emprendida de investigación, recopilación y catalogación de dichos espacios, se deduce una verdad incuestionable y es que, sin profundizar más y en términos exclusivamente cuantitativos, fueron muchas las estancias capitulares o auxiliares que se construyeron en las catedrales españolas durante los siglos del barroco. Entre todo ese gran volumen de edificaciones realizadas, son numerosas las que destacan significativamente por su alta calidad e interés, aunque también es cierto que hay un porcentaje considerable que presenta una muy discreta calidad en lo que concierne a los aspectos artísticos.

Aunque constituyen el conjunto de edificaciones que son indispensables para el funcionamiento de la sede en sus diversos aspectos, no se configuran exclusivamente como una mera arquitectura funcional, sino que en muchos de estos ámbitos, fundamentalmente en las sacristías y salas capitulares, se concentra todo un despliegue ornamental que les confiere una apariencia de gran riqueza, dignidad y suntuosidad.

En lo referente a la organización y disposición que presenta el conjunto de estas estancias en el edificio catedralicio, se ha observado que la zona más habitual para su ubicación suele estar en torno al claustro o, en el caso de que éste no exista, es frecuente que se dispongan en las proximidades de la cabecera, con la sacristía sirviendo de nexo con el templo. Pero a pesar de ello, se ha podido comprobar que no ha existido un único modelo de distribución a seguir, sino que las distintas fórmulas que se presentan vienen a ser tan diversas como lo son las diócesis y, en ocasiones, sus emplazamientos únicamente se explican porque algunos de los recintos actuales están sucediendo a construcciones anteriores.

De igual forma, se ha advertido el hecho de que no todos los conjuntos capitulares de las catedrales españolas pertenecientes a la época barroca se concibieron de un modo unitario y, por lo tanto, con sus distintas estancias dispuestas conforme a un criterio y orden preestablecidos. Se puede decir así que fueron varios los complejos de este tipo cuyas edificaciones quedaron organizadas de un modo que podríamos considerar orgánico, dado que se encuentran formados por sucesiones de unidades espaciales independientes, las cuales se iban añadiendo o readaptando en diversos momentos y en función de las distintas necesidades surgidas.

Además de las oportunas motivaciones estéticas o artísticas que incitarían a las corporaciones capitulares a emprender nuevos proyectos edificativos, se ha considerado que hubo una confluencia de diversos factores que propiciaron el auge constructivo de estos espacios durante el barroco, así como la remodelación o modernización de los que existían previamente. Entre ellos se puede destacar el aumento del número del personal catedralicio que se registró por aquel entonces, es decir, de las personas 
encargadas de alguna función en el cabildo, ya fuesen quehaceres religiosos, sociales, económicos, administrativos, benéficos, etc. Esta situación se mantuvo generalmente in crescendo hasta que, a partir del Concordato de 1851, se produjo un cambio drástico, reduciéndose de una manera muy considerable el número de personas vinculadas laboralmente a las distintas catedrales españolas.

Durante la Edad Moderna se experimentaron importantes cambios en el seno de las corporaciones capitulares. Probablemente el más significativo fuese el definitivo abandono de la vida comunitaria de sus miembros, ${ }^{4}$ motivo por el cual pudieron proceder a la renovación y adaptación para otros fines de los espacios que habían sido destinados a ello, como los antiguos dormitorios, la enfermería o el refectorio. Otros cambios se reflejaron, por ejemplo, en las formas de llevar a cabo la piedad capitular, de tal modo que se fueron sustituyendo las antiguas comidas para pobres en los refectorios, por la entrega directa de limosnas en efectivo, de ropa, panes u otros alimentos.

Jugó además un papel fundamental en este sentido el creciente volumen de rentas que se comenzó a percibir en las catedrales por aquellos momentos, lo que vino unido a una mayor complejidad en su sistema de recaudación, haciendo necesarios así nuevos espacios para su conveniente administración, registro y control.

En este desarrollo también tuvieron su repercusión los nuevos conceptos del rito y del ceremonial que se fueron implantando durante la Contrarreforma, en cumplimiento de las respectivas prescripciones tridentinas, a partir de los cuales se sucedieron numerosas actuaciones encaminadas a adaptar los antiguos edificios religiosos a las nuevas prácticas. Por aquel entonces adquirieron una gran relevancia los lugares destinados a contener en su interior los objetos de uso litúrgico, ya que éstos se iban incrementando y enriqueciendo progresivamente. De esta suerte, se incorporaron nuevos elementos al ajuar litúrgico de la catedral y algunos de los que hasta entonces eran simplemente funcionales, pasaron a ser sustituidos por verdaderos objetos suntuarios confeccionados con metales preciosos y otros ricos materiales. Por ese mismo motivo se hizo recomendable su custodia y conservación en lugares que resultasen suficientemente seguros y decorosos, incluyendo, si era necesario, nuevas edificaciones para ello.

Además de todo esto, cabe señalar la circunstancia de que algunas catedrales no habían sido dotadas en un principio de este tipo de ámbitos funcionales o, en cambio, lo habían hecho de modo provisional. Por lo tanto, durante la época barroca se pudo atender ya a las distintas necesidades espaciales que habrían ido surgiendo con el

4 Algunas catedrales españolas mantuvieron hasta fechas muy avanzadas el sistema de vida en comunidad de sus capitulares. El ejemplo más significativo es, sin duda alguna, el de la catedral de Pamplona, en la que dicho sistema no fue abolido hasta la segunda mitad del siglo XIX. 
tiempo en este sentido, una vez que hubiese sido concluida la edificación de la parte primordial del conjunto, esto es, la zona de culto.

Pero también se daba otra posibilidad y es que, ya por los siglos XVII y XVIII, algunas de las corporaciones capitulares llegaron a mostrar una preocupación muy justificada ante las condiciones que presentaban sus fábricas catedralicias y la estabilidad de las mismas, muchas de las cuales correspondían al periodo medieval, con lo que, en determinados casos, ya se habían comenzado a resentir por el paso del tiempo. Teniendo además en cuenta que los sectores destinados al servicio de la catedral solían presentar frecuentemente un carácter más efímero por la propia naturaleza de sus materiales, se entiende que fueran numerosas las nuevas empresas constructivas y reparadoras que se hizo necesario acometer por este motivo.

Asimismo, se ha podido comprobar que en muchas ocasiones la construcción de los nuevos espacios anexos al templo y la modernización o barroquización de los previos, se hizo posible gracias a las generosas iniciativas particulares de algunos obispos o también de miembros destacados del cabildo, propiciando empresas de alta calidad artística en sus respectivas sedes, para las que no dudaron además en requerir los servicios de algunos de los maestros más destacados del momento. 